ORIGINAL ARTICLE

\title{
The effect of sports specific training on reducing the incidence of hamstring injuries in professional Australian Rules football players
}

\author{
G M Verrall, J P Slavotinek, P G Barnes
}

Br J Sports Med 2005;39:363-368. doi: 10.1136/bjsm.2005.018697

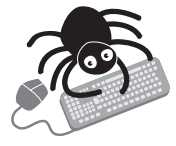

Video presentations of two examples of an injury being sustained can be viewed (MPEG 1 and 2) at http://www.bisportsmed. com/supplemental.

See end of article for authors' affiliations

.....................

Correspondence to: Geoffrey M Verrall, SPORTSMED.SA

Adelaide, Australia; verrallg@bigpond.com

Accepted 22 March 2005
Objectives: To assess in a single team of Australian Rules football players the effect of a specific intervention program on the incidence and consequence of hamstring muscle strain injuries.

Method: A prospective study was performed with a single team being followed for four playing seasons for hamstring injury. Magnetic resonance imaging was used to confirm the diagnosis of hamstring muscle injury. After two playing seasons an intervention program was implemented with the number of athletes with hamstring injury, competition days missed, and incidence of hamstring match injuries per $1000 \mathrm{~h}$ of playing time being compared pre- and post-intervention. The intervention program involved stretching whilst fatigued, sport specific training drills, and an emphasis on increasing the amount of high intensity anaerobic interval training.

Results: In the seasons prior to the intervention, nine and 11 athletes sustained hamstring injury compared to two and four following intervention. Competition days missed reduced from 31 and 38 to 5 and 16 following intervention and match incidence decreased from 4.7 to 1.3 per $1000 \mathrm{~h}$ of playing time. A beneficial effect was demonstrated with a smaller number of players having hamstring injuries $(p=0.05)$, a lower number of competition games missed being recorded $(p<0.001)$, and a decrease in hamstring strain incidence per $1000 \mathrm{~h}$ of playing time $(p=0.01)$ following the intervention program.

Conclusions: Increasing the amount of anaerobic interval training, stretching whilst the muscle is fatigued, and implementing sport specific training drills resulted in a significant reduction in the number and consequences of hamstring muscle strain injuries.
A lthough hamstring muscle strain injury is common in many sports, there is little in the literature concerning prevention of this injury. ${ }^{12}$ Previous studies have focused on increasing hamstring strength ${ }^{3-6}$ as a means of preventing hamstring injury. However, it has been conceded that well designed prospective hamstring injury prevention studies are lacking. ${ }^{1} 7$

Australian Rules football has a high incidence of hamstring injury. ${ }^{8}$ This is due, at least in part, to the nature of the game with athletes undertaking intensive repeated sprinting efforts over a relatively prolonged period. In this respect, the hamstring muscle demands in Australian football are similar to those in soccer, another sport with a high hamstring strain injury rate. ${ }^{9-11}$ As a consequence of these high hamstring injury rates, athletes participating in these sports are ideal to assess the effects of any hamstring injury prevention program.

Accordingly, the aim of this study was, in a group of Australian football players, to assess the effect of an intervention program on the number of athletes having, and the consequences of (competition games missed), hamstring muscle strain injuries.

\section{METHODS \\ Subjects}

One professional Australian Rules football team playing in a state based competition consented to be involved in this four season study. The team had a yearly total squad of 70 players (range 69-71) that played in weekly competition matches. Including pre-season and play off matches, the team averaged 24 games (range 23-26) in each of the playing seasons.

\section{Injury definition}

A hamstring muscle injury was defined as an athlete having posterior thigh pain, where direct contact with the thigh was excluded as a cause of the injury, with subsequent detection of hyperintense signals within the hamstring muscle(s) on magnetic resonance imaging (MRI) T2-weighted fat suppressed images. The MRI was independently reported by a musculoskeletal radiologist (JPS) with only the presence or absence of hamstring injury being reported to the team physician (GMV). This MRI criterion for hamstring muscle injury detection has been used in prior studies. ${ }^{12}{ }^{13}$ Athletes with a posterior thigh injury and a negative MRI scan for hamstring muscle strain injury were also recorded.

In our study, all hamstring injuries resulted in missed match playing time. Therefore, our injury definition was the same as that used for the national competition Australian Football League (AFL) injury surveillance database. ${ }^{8}$

\section{Outcome measures}

In each of the four playing seasons, the number of athletes injured and the number of competition games missed was recorded for hamstring muscle strain injuries. Injury incidence was also calculated for match and training injuries, expressed per 1000 player hours for match injuries and per 1000 player weeks for training injuries. Recurrent injuries within the same season were not included in these calculations. All of these measures were compared with the AFL injury database $\mathrm{e}^{8}$ over the identical time period.

Other information recorded throughout the study period included player turnover (the number of players transferring

Abbreviations: AFL, Australian Football League; MRI, magnetic resonance imaging 
into and out of the squad each season), the total number of injury episodes (to account for athletes having injury recurrence), the number of athletes injured without a prior history of hamstring injury, and the convalescent interval after injury. We used these other measures to determine whether any confounding factors may have been responsible for any intervention effect achieved.

\section{All years}

In the pre-season period (approximately 16-20 weeks in duration) athletes undertake extensive aerobic and anaerobic interval training, under supervision from the fitness coach, to prepare for the forthcoming playing season. Before each training session in all the studied years, the players had a supervised warm up session. Generally this commenced with a slow run of approximately $800 \mathrm{~m}$ followed by running exercises, using an incremental increase in running speed for each repetition, of 10 repetitions of $60 \mathrm{~m}$. Generalised warm up stretches of the lower limbs were also performed in this warm up period during the break after each running repetition.

\section{Designing the prevention program}

During the first 2 years of the pre-intervention period (years 1 and 2) the following observations with respect to the ongoing training program and the nature of hamstring injuries in Australian football were made:

1. Emphasis in the pre-season training period was on aerobic conditioning with 3 and $5 \mathrm{~km}$ running time trials being used to monitor player fitness levels.

2. There was no specific control of, or instructions given on, the stretching or weights program regimes undertaken by players.

3. Analysis of hamstring injuries in Australian Rules football players demonstrated that most injuries occur after the athlete has been playing for a significant amount of time, suggesting fatigue is a risk factor for hamstring injury. ${ }^{14}$

4. The majority of hamstring strain injuries in Australian football have been noted to occur in match playing situations, as opposed to training sessions, ${ }^{14}$ and occur during running and acceleration. ${ }^{14}$

5. Video analysis of many hamstring injuries (authors' unpublished observations) in Australian football suggests that many of these injuries are sustained with the athlete flexing at the trunk whilst running just prior to the injury occurring. This act of trunk flexion is usually in the process of acceleration and/or in attempting to reach down to grasp the ball whilst running at high speed. A stride by stride motion of an injury can be seen in fig 1. Video presentations of two examples can be viewed (MPEG 1 and 2) at http://www.bjsportsmed. com/supplemental. MPEG 1: The athlete chasing the ball is running and in the process of grabbing the ball bends at the trunk and suffers a subsequent hamstring injury. MPEG 2: The athlete is leading towards the ball and in the process of grasping the ball bends at his trunk whilst sprinting and suffers a subsequent hamstring injury. This MPEG is demonstrated in slow motion. Informed written consent has been obtained from the injured athletes identified in these videos.

\section{Intervention program - years 3 and 4}

From these observations the following program, undertaken by all players, was commenced in the pre-season conditioning period of the third year of injury surveillance.
1. More high intensity anaerobic interval running/acceleration drills that were designed to more accurately reflect the match playing situation were implemented in the pre-season conditioning period. This philosophy became the guiding principle for most, if not all, preand in-season training exercises. Player fitness was monitored by multi stage fitness testing (20 m repeated shuttle run test) with middle distance running trials no longer being used.

2. The players were encouraged to perform hamstring stretches (passive isometric) during breaks in playing or training. These were also made compulsory after training or playing had ceased. It was intended that these exercises be performed at times when the athletes were considered to have some degree of muscle fatigue. Stretches used for this part of the program consisted of a single posterior thigh stretch with the leg in varying degrees of knee flexion $\left(0^{\circ}, 10^{\circ}\right.$, and $\left.90^{\circ}\right)$ using either the ground, perimeter fence, or a table (depending on circumstances) to stabilise the tested leg. Subsequent flexion of the trunk was used to stretch the hamstring muscles. The athletes were encouraged to hold the stretch for at least $15 \mathrm{~s}$ and to rotate the actual stretch performed, by using varying degrees of knee flexion, throughout the course of the training or playing session.

3. A specific football training drill was designed where the athlete would consistently change running speed with the body positioned in trunk flexion. This drill involved a player tapping and controlling a rolling football (Australian football has an oval shaped ball) in front of them whilst an opponent attempted to take over, from behind the athlete, the position of being the athlete in control of the ball. Thus both athletes are in a position of trunk flexion for the exercise. This exercise was performed for 5 min twice a week at the end of a training period.

4. Instructions with regards to weight training of the lower limb were given. Those athletes who had not previously used leg weights were told to not commence using them. Those who had used leg weights in the past were specifically told to avoid open chain exercises on weights machines. Furthermore, if they were to do leg weights they were to be supervised directly by the fitness coach in closed chain leg weight exercises. A total of eight athletes undertook closed chain leg weights at some stage during the intervention period.

\section{Outcome measures and statistical analysis}

The results from the first 2 years of the injury surveillance were compared to the results from the 2 years after implementation of the intervention program. To determine whether an intervention effect was present, Poisson regression analysis was performed. A p level of less than 0.05 was considered to indicate statistical significance.

\section{RESULTS}

Table 1 lists outcome measures comparing the single team with AFL data. Our calculated match play injury incidence for the single team was 4.7 per 1000 playing hours in the preintervention period, dropping to 1.3 injuries per 1000 playing hours after intervention. Training injuries decreased from 1.7 per 1000 player weeks to 0.7 per 1000 player weeks postintervention.

Poisson regression analysis demonstrated an intervention effect in the 2 years following the introduction of the intervention program, with the studied team having both fewer players with hamstring injury $(Z=2.01, p<0.045)$ and fewer competition games missed due to these injuries 

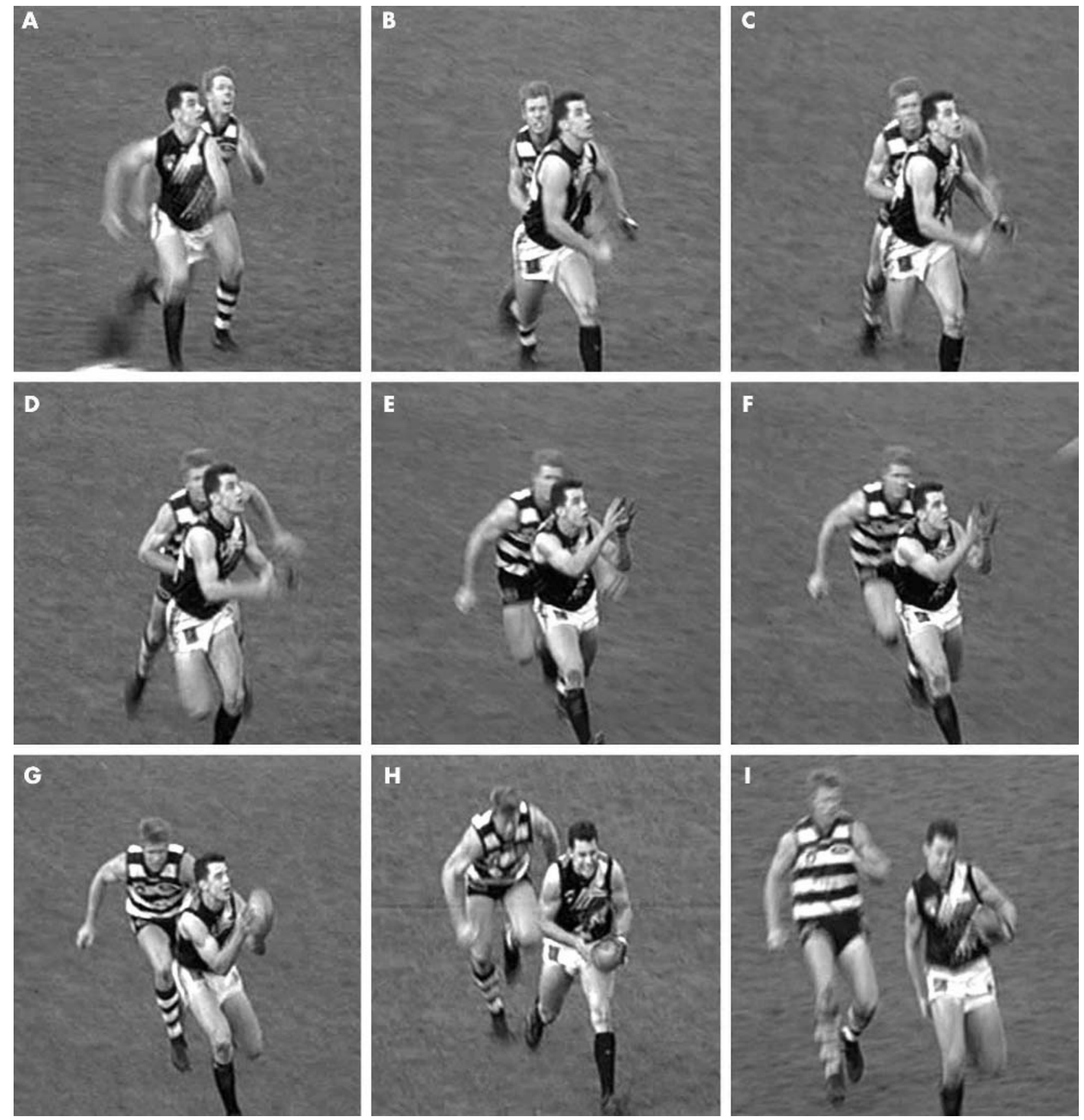

Figure 1 Still pictures from MPEG 2. Image A: Stride 1: Ground contact R foot - erect body. Image B: Stride 2: Ground contact L foot - trunk flexion. Image C: Stride 3: Early swing R leg - further trunk flexion. Image D: Stride 3: Mid swing R leg - further trunk flexion. Image E: Stride 3: Full extension R leg - trunk flexion - full extent. Image F: Stride 3: Ground contact R leg - ball arrives. Image G: Stride 4: Mid swing L leg - pain on face. Image H: Stride 4: Ground contact L leg - pain on face. Image I: Stride 5: Ground contact R leg - grasps R hamstring. Informed written consent has been obtained from the athletes identified in this figure.

$(\mathrm{Z}=4.12, \mathrm{p}<0.001)$. Incidence calculations demonstrated an intervention effect with a decrease in the incidence of hamstring strain in matches $(\mathrm{RR}=0.267$, confidence interval (CI) 0.076 to $0.764, p=0.012$ ). However, the training incidence decrease in hamstring injuries was not statistically significant $(\mathrm{RR}=0.400$, CI 0.054 to $2.028, \mathrm{p}=0.29)$.

In each year of the study, 16 (23\%), 15 (22\%), 18 (25\%), and $15(21 \%)$ of athletes, respectively, had a prior history of hamstring injury. Player turnover averaged 13\% (10 athletes, range 8-14) and was similar for each playing season. The mean number of competition days missed throughout the study period for all hamstring injuries was 25 days per injury in each season (range 23-26 days). The results for other potential confounding factors for the single team are given in table 2 .

\section{DISCUSSION}

\section{Principal finding and study weaknesses}

The principal finding of this study was that implementation of a specific intervention program resulted in a significant reduction in the number, incidence, and consequences of (competition games missed) hamstring injuries in a population of Australian football players. This intervention program emphasised increasing the amount of high intensity 
Table 1 Number of games missed and number of players injured (single team and AFL national competition)

\begin{tabular}{lcccc}
\hline & Year 1 & Year 2 & Year 3 & Year 4 \\
\hline Games missed (number) & & & & \\
$\quad$ Single team & 31 & 38 & 5 & 16 \\
$\quad$ AFL teams average & 36 & 37 & 39 & 37 \\
Players injured (number) & & 11 & 2 & 4 \\
$\quad$ Single team & 9 & 11 & 10 & 11 \\
$\quad$ AFL teams average & 11 & 12 & 1.3 \\
Match incidence (single team) & 4.4 & 5.0 & 1.3 & 1.3 \\
Training incidence & 1.4 & 2.2 & 0 & 1.4 \\
(single team) & & & & \\
\hline
\end{tabular}

The AFL lists of players $(42,42,41$, and 43 players, respectively, for the study period) have been converted to a 70 player list. Match incidence is per 1000 player hours, training incidence is per 1000 player weeks. The AFL average for the same time period is match incidence 4.3 , training incidence 2.2.

anaerobic interval training (simulating the running requirements of the sport itself). It also involved development of a specific football training exercise undertaken with the athlete in a position of trunk flexion and encouraged the athlete to undertake passive isometric stretching exercises with the hamstring muscle in a relatively fatigued state.

It is not possible to account for all the components of a preand in-season training program nor is it possible to supervise the off field training program of athletes. Therefore, we cannot be confident as to which component of our intervention program, if any, may have been responsible for the key findings in this study. This, along with our relatively small athlete numbers and the use of historical controls are the principal limitations to interpretation of the findings in this study.

\section{Rationale of the intervention program}

Role of training/playing specificity in muscle strain injuries

The intervention program was designed to change the emphasis of pre-season conditioning and in-season training from predominantly aerobic training to increased high intensity interval anaerobic training. In effect, we were attempting to change the specificity of training to "training as the game is played". This should help prevent hamstring injury by improving hamstring muscle conditioning and developing increased fatigue resistance. It was considered this would enable the athlete to better cope with the running stressors encountered during the game situation, the principal time for hamstring injury.

For a similar reason our program also had a component of running/accelerating in a position with increased trunk flexion.

\section{Role of fatigue in muscle strain injuries}

In the laboratory, fatigued muscles are able to absorb less energy when undergoing stretching, thus making them more vulnerable to injury when compared to non-fatigued muscles. $^{2}{ }^{15}$ Therefore, improving fatigue resistance in muscle should help prevent muscle strain injury.

\section{Role of stretching in preventing hamstring muscle} strain injuries

In the clinical situation, the effect of stretching on hamstring injury prevention is not conclusive. ${ }^{16}{ }^{17}$ Despite this, we considered that stretching could have a role in hamstring muscle strain injury prevention by improving force absorption for a given length of muscle, thereby making the muscle
Table 2 Results from single team for the four studied seasons

\begin{tabular}{lllll}
\hline & Year 1 & Year 2 & Year 3 & Year 4 \\
\hline $\begin{array}{l}\text { Number of injury episodes } \\
\text { (recurrence rate in brackets) }\end{array}$ & $12(33 \%)$ & $15(27 \%)^{*}$ & $2(0 \%)$ & $6(50 \%)$ \\
$\begin{array}{l}\text { Number injured without past } \\
\text { history }\end{array}$ & 6 & 0 & 2 \\
$\begin{array}{l}\text { Athletes with MRI negative } \\
\text { injuries }\end{array}$ & 2 & 3 & 2 & 2 \\
\hline *One player had three episodes of injury. \\
$\begin{array}{l}\text { The number of injury episodes includes the original injury for that season } \\
\text { and recurrent injury. So, for the first year, nine players had an injury with } \\
\text { three of these having a recurrent injury in that season for a total of } 12 \\
\text { injury episodes. }\end{array}$
\end{tabular}

more resistant to stretch injury. This is extrapolated from the well understood principle that the viscoelasticity properties of muscle can be changed by stretching, with studies demonstrating that stretching results in a reduction in load on the muscle-tendon unit for any given length. ${ }^{218}$ In our prevention program, we implemented the stretching regime by a combination of encouragement, demonstration, and compulsion. We principally performed these stretches at a time when it was considered that the muscle would be fatigued.

\section{Weights program}

A weights program is undertaken by all players in professional Australian football. In our single studied team, this program targets the upper body with lower limb weights, either open or closed chain, not being an integral part of the program.

As we wanted to target running and stretching as the key intervention features of our prevention program, we specifically requested that athletes avoid the use of leg weights as we recognised that this could be a major potential confounding factor. We believe that the majority of athletes acceded to our request not to do leg weights and that no one undertook open chain weights during the study period. We consider this approach can be justified by the nature of our demonstrated results, but we cannot comment on how a lower limb weights program may influence the incidence of hamstring injuries.

\section{Key features of a hamstring injury prevention intervention program}

We believe the key features of any hamstring injury prevention program in sports such as Australian football with its requirement for much high intensity anaerobic interval running to be as follows: (1) to improve the training regime so that it more accurately reflects match playing conditions with the goal of improving muscle conditioning; (2) to try and improve fatigue resistance of the hamstring muscle; and (3) to try and induce a change in the viscoelastic properties of muscle so as to increase energy absorption and decrease load on the muscle-tendon unit for any given length especially in body positions of function and vulnerability to injury.

\section{Comparison to other studies}

It has been demonstrated that pre-season conditioning with sport specific exercises decreases lower limb injury in soccer, although this study was not specific for hamstring injuries. ${ }^{19}$ Another recently published study ${ }^{3}$ involved athletes increasing eccentric strength by use of an open chain weight machine. The pre-season strengthening program in this study decreased the number of hamstring injuries when compared to those athletes who did not undertake pre-season strengthening. However, the clinical application of this study 
is limited as the positive effect (decrease in the number of hamstring injuries) was only demonstrated in minor injuries (athlete missing less than a week of training/playing) with the more troublesome clinically moderate injuries (athlete missing more than a week but less than a month) demonstrating no significant benefit.

Other published hamstring prevention studies had significant methodological flaws including retrospectivity, the absence of comparison groups, and inadequate injury definitions. ${ }^{45}$

\section{Validity of this study}

This study involved a single state based competition team and the injuries sustained were compared to the AFL injury database. ${ }^{8}$ It has been demonstrated previously that there is no significant difference in the hamstring injury rates between state and national competitions..$^{20}$ Our pre-intervention exposure data demonstrated a similar injury incidence (4.7 injuries per 1000 playing hours) when compared to the AFL injury database (4.3 per 1000 playing hours ${ }^{8}$ ). This compares to elite soccer with 3.0 hamstring injuries per 1000 playing hours. ${ }^{21}$ By simulating match playing conditions during training sessions, we were able to significantly decrease the incidence of match playing injuries $(p=0.01)$. Consistent with this approach, we did not see a statistical reduction in the incidence of training injuries $(p=0.29)$.

As the number of athletes with posterior thigh injuries without MRI evidence of hamstring injury was similar in all studied seasons, the reduction in the number of games missed is not due to a differing classification of posterior thigh injuries. The proposed aetiology of these posterior thigh injuries without MRI evidence of injury has been discussed previously. ${ }^{14} 2022$ Other potential confounding factors did not demonstrate any difference in any of the studied seasons. The cohort remained reasonably stable over the studied period with actual turnover being $13 \%$ each season and the number players having prior hamstring injury, a major risk factor for injury, ${ }^{20}{ }^{21}$ remaining constant $(23-25 \%)$ in each season.

This study was performed over an extended time period to avoid results that may have occurred as a consequence of a single "lucky" season with respect to the number of hamstring injuries. Also, as many of the potential confounding factors have been accounted for, we believe the decrease in the number of hamstring injuries and subsequent competition games missed is a true intervention effect.

Although all the specific components of our intervention program had a potential benefit in decreasing the hamstring injury rate, we do not believe a single intervention exercise in isolation was the reason for the beneficial effect. Rather a change of philosophy to "training as the game is played" was considered to be the principal factor responsible for the reduction in the number of hamstring injuries demonstrated in this study. As the implemented program is relatively simple in scope and concept, it should be transferable to other Australian football teams as well as to other sports with high intensity interval running, although this remains an area for future research.

\section{CONCLUSIONS}

An intervention program based on increased high intensity interval anaerobic training that more accurately reflected match playing conditions, stretching whilst the muscle was fatigued, and the implementation of sport specific training drills was implemented. This resulted in a significant reduction in the number of hamstring muscle strain injuries and consequently in the number of competition games missed in a group of Australian football players.
What is already known on this topic

Although hamstring injuries are common, very little exists in the literature on the prevention of these injuries in sport. Studies that have been performed have concentrated on improving muscular strength as a means of preventing hamstring injury.

\section{What this study adds}

This study adds to the current knowledge of hamstring injury prevention by demonstrating that the incidence and consequence of these injuries can be reduced by undertaking a training program that more accurately reflects the conditions where the majority of injuries occur, that is, during match play, and by doing specific exercises.

\section{ACKNOWLEDGEMENTS}

The authors wish to thank Dr Adrian Esterman for his statistical analysis and the Norwood Football Club for their participation in this study.

\section{Authors' affiliations}

G M Verrall, P G Barnes, SPORTSMED.SA, Adelaide, Australia J P Slavotinek, Department of Medical Imaging, Flinders Medical Centre, Adelaide, Australia

The authors received no financial incentive from being involved in this project.

\section{Competing interests: none declared}

Informed written consent has been obtained from the athletes identified in the illustrations used in this manuscript.

\section{REFERENCES}

1 Bahr R, Holme I. Risk factors for sports injuries - a methodological approach. Br J Sports Med 2003;37(5):384-92.

2 Garrett WE. Muscle strain injuries. Am J Sports Med 1996;24(6):S2-8.

3 Askling C, Karlsson J, Thorstensson A. Hamstring injury occurrence in elite soccer players after pre-season strength training with eccentric overload. Scand J Sci Med Sports 2003;13:244-50.

4 Dornan P. A report on 140 hamstring injuries. Aust J Sci Med Sport 1971:4(2):30-6.

5 Heiser TM, Weber J, Sullivan G, et al. Propylaxis and management of hamstring muscle injuries in intercollegiate football players. Am J Sports Med 1994;12(5):262-6.

6 Miolsnes R, Arnason A, Osthagen T, et al. A 10 week randomized trial comparing eccentric vs. concentric hamstring strength in well-trained soccer players. Scand J Sci Med Sports 2004; 14(5):31-7.

7 Knapnik JJ, Jones B, Bauman C, et al. Strength, flexibility and athletic injuries. Sports Med 1992;14:277-88.

8 Orchard J, Seward H. Epidemiology of injuries in the Australian Football League, seasons 1997-2000. Br J Sports Med 2002;36(1):39-45.

9 Anderson TE, Tenga A, Engebretsen, et al. Video analysis of injuries and incidents in Norwegian professional football. Br J Sports Med 2004;38(5):626-31

10 Arnason A, Gudmundsson A, Dahl HA, et al. Soccer injuries in Iceland. Scand J Med Sci Sports 1996;6:40-5.

11 Hawkins RD, Fuller CW. A prospective epidemiological study of injuries in four English professional football clubs. Br J Sports Med 1999;33:196-203.

12 Pomeranz S, Heidt R. MR imaging in the prognostication of hamstring injury. Radiology 1993;189:897-900.

13 Slavotinek JP, Verrall GM, Fon GT. Hamstring injury in athletes: the association between MR measurements of the extent of muscle injury and the amount of time lost from competition. AJR Am J Roentgenol 2002;179:1621-8

14 Verrall GM, Slavotinek, Barnes PG, et al. Diagnostic and prognostic value of clinical findings in 83 athletes with posterior thigh injuries. Comparison of clinical findings with magnetic resonance imaging documentation of hamstring muscle strain. Am J Sports Med 2003;31(6):969-73.

15 Mair S, Seaber A, Glisson R, et al. The role of fatigue in susceptibility to acute muscle strain injury. Am J Sports Med 1996;24(2):137-43.

16 Ekstrand J, Gillquist J. The frequency of muscle tightness and injuries in soccer players. Am J Sports Med 1982;10:75-8. 
17 Worrell TW Perrin DH, Gansneder BM, et al. Comparison of isokinetic strength and flexibility measures between hamstring injured and noninjured athletes. J Orthop Sports Phys Ther 1991;13:118-25.

18 Taylor DC, Dalton JD, Seaber AV, et al. Experimental muscle strain injury. Early functional and structural deficits and the increased risk for reinjury. Am J Sports Med 1993;21:190-4.

19 Heidt RS, Sweeterman LM, Carlonas RL, et al. Avoidance of soccer injuries with pre-season conditioning. Am J Sports Med 2000;28:659-62.
20 Verrall GM, Slavotinek JP, Barnes PG, et al. Clinical risk factors for hamstring muscle strain injury: a prospective study with correlation of injury by magnetic resonance imaging. Br J Sports Med 2001;35(6):435-40.

21 Arnasson A, Sigurdsson S, Gudmundsson A, et al. Risk factors for injuries in football. Am J Sports Med 2004;32(1S):5S-16S.

22 Gibbs N, Cross T, Cameron M, et al. The accuracy of MRI in predicting recovery and recurrence of acute grade one hamstring strains in Australian Rules football players. J Sci Med Sport 2004;7(2):248-58.

\section{bmjupdates+}

bmjupdates+ is a unique and free alerting service, designed to keep you up to date with the medical literature that is truly important to your practice.

bmjupdates+ will alert you to important new research and will provide you with the best new evidence concerning important advances in health care, tailored to your medical interests and time demands.

Where does the information come from?

bmiupdates+ applies an expert critical appraisal filter to over 100 top medical journals A panel of over 2000 physicians find the few 'must read' studies for each area of clinical interest

Sign up to receive your tailored email alerts, searching access and more...

www.bmjupdates.com 